\title{
Cogging Torque Reduction of BLDC Motor using Level Set Based Topology Optimization Incorporating with Triangular Finite Element
}

\author{
Xiangjun MENG ${ }^{\mathrm{a}}$, Shuhong $\mathrm{WANG}^{\mathrm{a}}$, Jie QIU' ${ }^{\mathrm{a}}$, Jian Guo ZHU ${ }^{\mathrm{b}}$, Youguang GUO \\ ${ }^{a}$ Faculty of Electrical Engineering, Xi'an Jiaotong Univerisity, Xi'an 710049, China \\ ${ }^{b}$ School of Electrical, Mechanical and Mechatronic Systems, University of Technology, Sydney, \\ NSW 2007, Australia
}

\begin{abstract}
Cogging torque in permanent-magnet brushless DC (BLDC) motor causes torque and speed ripples, as well as acoustic noise and vibration, especially in low speed and direct drive applications. In this paper, the stator core shapes are optimized by using a level set based topology optimization to reduced cogging torque. The level set method can represent the precise boundary shape of structure and also deal with complex topologies changes during the optimization process. Different from the conventional rectangular meshes, the level set technique based triangular finite element meshes is presented in this paper to deal with irregular domains. The comparison of cogging torque calculated before and after topology optimization verifies the advantages of level set topology optimization based on triangular meshes.
\end{abstract}

Keywords. Topology optimization, level set, adjoint variable, cogging torque

\section{Introduction}

The permanent magnet BLDC motor is required running in a low vibration and acoustic noise in many industrial applications. The cogging torque is one of the main causes which produce motor vibration. The cogging torque is caused by the magnetic field interaction between the permanent magnet on the rotor and the stator due to the slotting. In order to reduce the cogging torque, several ways, such optimize the stator pole shape or the rotor surface, can be used.

Topology optimization (TO) technique has been used in the optimization of material distribution in mechanical structure design [1]. Element based density method, one type of topology optimization methods, often result in mesh-dependent boundaries and grayscale elements due to numerical instabilities. Semyung Wang[1, 2] applied the continuum design sensitivity method in topology optimization of induction motors and DC motor. Dong-hun Kim[3] developed Smooth Boundary Topology Optimization in electrostatic problem. Takahashi [4] optimize the topology of magnetic record by using ON/OFF method. The level set based topology optimizations can provide smoother optimal boundaries and material interfaces; avoid the grayscale elements using density method. However, in the case of electromagnetic systems, this technique currently focuses on the application in complex devices, such as actuators, electrical machines, MRI, and so on. The triangular finite element type approximation is desired because the domains are irregular and interfaces on manifolds in complicated devices [5].

In this paper, the level set topology optimization based on triangular meshing is adopted in the design of stator core shape to minimize the cogging torque of BLDC motor.

\section{Level set function}

The level set based topology optimization is to represent design domains and the material boundaries by continuum level set function. Comparison with the parameterization, Level set is given as an implicit equation of a high dimension function [5]. By solving the level set function, the optimized fitness function is obtained. 
For a given domain $\Omega(t)$ and the boundary $\partial \Omega$, which is shown as Fig. 1, the implicit function $\phi(X)$ is defined

$$
\begin{cases}\phi(x, t)>0 & \text { in } \Omega(t) \\ \phi(x, t)=0 & \text { on } \partial \Omega \\ \phi(x, t)<0 & \text { in } R^{n} \backslash \bar{\Omega}(t)\end{cases}
$$

Where, the zero level set $\phi(X)=0$ describe the materials boundaries of the domain $\Omega(t)$. In the case of electrical machine, the domain $\Omega(t)$ may be applied to describe the stator and $\phi(X)=0$ distinguish the material boundaries between the stator core pole and the air gap.



Figure 1: Material boundary representation with level set function

Introduce a pseudo-time $t$ [6] for the dynamic process of the boundary propagation, one can obtain the following first order Hamilton-Jacobi equation:

$$
\frac{d \phi(x(t), t)}{d t}=\frac{\partial \phi(x(t), t)}{\partial t}+V_{n}|\nabla \phi|=0
$$

where, $V_{n}$ is the outward normal velocity of the zero level set, which depends on the objective function. $V_{n}$ may be solved by using Adjoint Variable Method (AVM) based on shape function of triangular finite element.

In this paper, the shape boundary of stator tooth of a BLDC motor is implicitly represented through an embedded level set function and propagated along its normal direction with speed expressed in terms of the cogging torque and the boundaries of materials.

\section{Adjoint variable design sensitivity analysis}

Consider the system objective function may be written in integral form as

$$
\varphi=\int_{\Omega} g(A, p) d \Omega
$$

[7]

According to finite element method, the finite element matrix equation is written as

$$
K(p) A=J
$$

Using the chain rule of differentiation, the total derivative of $\varphi$ with respect to $p$ may be calculated as

$$
\frac{d \varphi}{d p}=\frac{\partial \varphi}{\partial p}+\frac{\partial \varphi}{\partial A} \frac{\partial A}{\partial p}
$$

Differentiating both side of Eq. (4) with respect to design vector $p$, 


$$
K(p) \frac{d A}{d p}=-\frac{\partial(K(p) A)}{\partial p}+\frac{\partial J}{\partial p}
$$

Substituting Eq. (6) into Eq. (5) to obtain the design sensitivity of the objective function with respect to design vector $p$

$$
\frac{d \varphi}{d p}=\frac{\partial \varphi}{\partial p}+\frac{\partial \varphi}{\partial A} K^{-1}(p) \frac{\partial}{\partial p}(J-K(p) A)=\frac{\partial \varphi}{\partial p}+\lambda^{\mathrm{T}} \frac{\partial}{\partial p}(J-K(p)
$$

Introduce adjoint variable $\lambda$ to avoid solving reverse matrix of $K^{-1}(p), \lambda$ is defined as

$$
\lambda=\left[\frac{\partial \varphi}{\partial A} K^{-1}(p)\right]^{\mathrm{T}}=K^{-1}(p) \frac{\partial \varphi^{\mathrm{T}}}{\partial A}
$$

And $\lambda$ may be obtained by solving the following adjoint equation

$$
K(p) \lambda=\frac{\partial \varphi^{\mathrm{T}}}{\partial A}
$$

Finally, the outward normal velocity of the zero level set may be wrriten as

$$
V_{n}=-\frac{d \varphi}{d p}=-\left(\frac{\partial \varphi}{\partial p}+\lambda^{\mathrm{T}} \frac{\partial}{\partial p}(J-K(p) A)\right)
$$

\section{Triangular finite element based topology optimization}

In this paper, a nonlinear magnetic static problem is considered for structural topology optimization (TO). The zero level set boundary (LSB) moves to next position by solving Eq. 2. The stiffness matrix of finite element method should be reconstructed due to the boundary modification of material. Two methods may be employed. One is remeshing, in which the parameterization techniques should be used. The Alternative method is to change the magnetic reluctivity, $v$, of the finite elements, which intersect the zero level set boundary. The discontinuous Heaviside function can be regularized as a smoothed and continuous form

$$
H(\phi(p))=\left\{\begin{array}{ll}
1, & \text { if } \phi \geq 0 \\
0, & \text { if } \phi<0
\end{array} \text { and } \delta(p)=H^{\prime}(\phi(p)) \begin{cases}=0 & \text { if } \phi \neq 0 \\
\neq 0 & \text { if } \phi=0\end{cases}\right.
$$

The magnetic reluctivity of the triangular finite elements which lies on the boundary will be considered as constant and can be adjusted as

$$
v^{e}=\left[\sum_{i=1}^{3}\left(H\left(\phi_{i}(p)\right)\right) / 3\right]^{n} v
$$

where $2 \leq n \leq 4$ is penertry coefficence same as typical SIPM approch [8]. Fig. 2 schematic shows the reluctivity adjustment of triangular finite elements which lie on the zero level set boundaries.

\section{Algorithm}

Generally, the basic procedure to the level set based topology optimization is shown in Fig.3. The convergence criterion is the maximum normal velocity of the level set boundary 
less than a given threshold, $\varepsilon$. The function $\phi(X)$ can be redefined in each iteration due to boundary propagation.

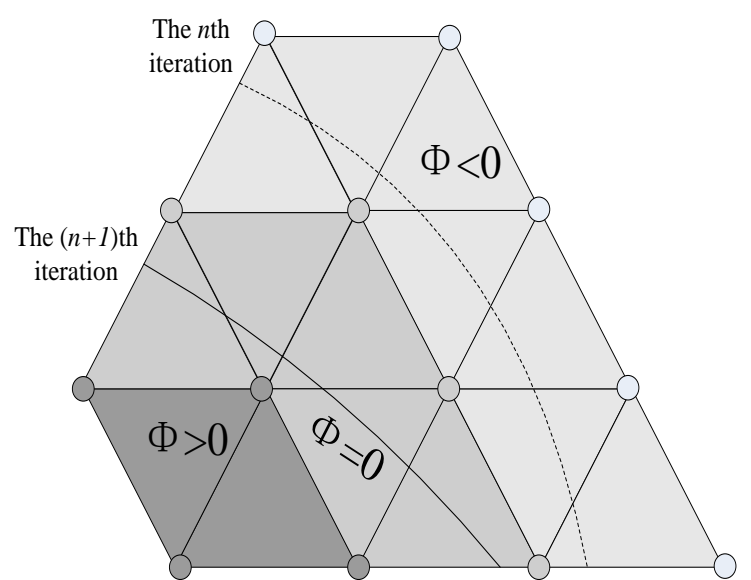

Figure 2: Schematic for LSB propagation

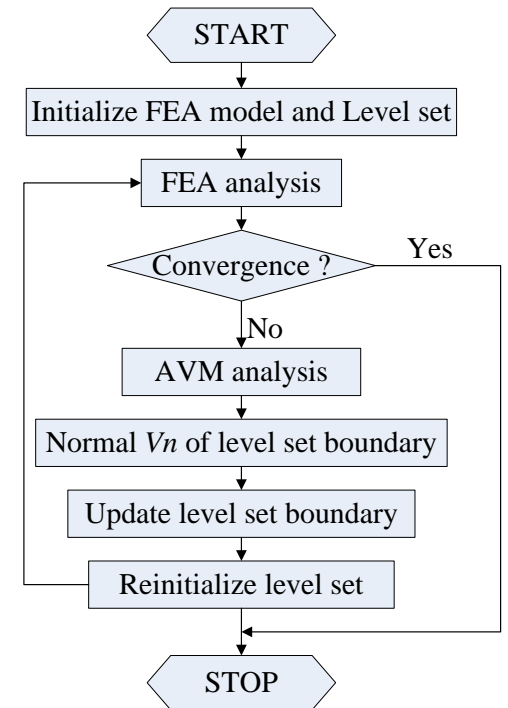

Figure 3: Flowchart for the level set based topology optimization

\section{Numerical example}

The algorithm of the level set based topology optimization is numetically implemented using 2-dimensional FEM coded in FORTRAN, and is applied to the opitimization of the BLDC motor stator pole shape to decrease the cogging torque.

Table I lists the specification of the BLDC motor and the cross-section structrue is shown in Fig. 4.

Table I

Specifications of the BLDC motor

\begin{tabular}{llll}
\hline Rated power $/ \mathrm{kW}$ & 0.8 & Length of airgap $/ \mathrm{mm}$ & 0.5 \\
Rated voltage/ $\mathrm{V}$ & 178.0 & Inner radius of rotor $/ \mathrm{mm}$ & 7.15 \\
Pole number & 6 & Outside radius of rotor $/ \mathrm{mm}$ & 29.0 \\
Slots number & 9 & Remanence $/ \mathrm{T}$ & 0.38 \\
Stator outside radius $/ \mathrm{mm}$ & 50.55 & Axial length of stack $/ \mathrm{mm}$ & 61.0 \\
\hline
\end{tabular}

The cogging torque, the object function of the optimization, is calculated by using Maxwell stress tensor method. The flux density in the airgap can not be lower than a given value. In general case, the topology problem of stator pole shape can be specified as[10] 


$$
\begin{array}{ll}
\text { Minimize } & \bar{T}_{c}(A, \phi)=\frac{1}{g} \sum_{i=1}^{N_{\text {airgap }}} R_{i} \frac{B_{r i} B_{t i}}{\mu_{0}} A_{i} H\left(\phi_{i}\right) \\
\text { subject to } & B_{r_{\text {airgap }}}>0.3 \mathrm{~T}
\end{array}
$$

where, $T_{c}$ is the cogging torque, $R_{i}$ is the distance beween the centers of the ith element and shaft, $B_{r i}$ and $B_{t i}$ are radial and tangential components of magnetic flux density of the $i$ th element in the airgap respectively, $g$ and $A_{i}$ are the length of airgap and area of the ith airgap element. $B r_{\text {airgap }}$ is the average radial component of flux density in airgap.

Differentiating both side of Eq. (14) with respect to system state variables, magnetic vector potential $A$

$$
\frac{\partial \bar{T}_{c}(A, \phi)}{\partial A}=\frac{1}{g} \sum_{i=1}^{N_{\text {airgap }}} \frac{R_{i} A_{i}}{\mu_{0}}\left(\frac{\partial B_{r i}}{\partial A} B_{t i}+B_{r i} \frac{\partial B_{t i}}{\partial A}\right) H\left(\phi_{i}\right)
$$

Substituting Eq. (15) into Eq. (9), the design sensitivity of the objective function with respect to design vector $v$, i.e. outward normal velocity of the zero level set boundary can be achieved.

The initial pole shape is shown in Fig. 5. The optimal shape of stator tooth using both density method and level set function are illustrated in Fig. 6 and 7, respectively. Comparison between the optimized results suggests that the level set based topology optimization can avoid the grayscale elements which often occur in density method and can provide a smooth boundaries.

Fig. 8 shown the value objective function with respect to optimization iteration. The maximum of cogging torque is decreased from initial value $0.09 \mathrm{~N} . \mathrm{m}$ to $0.024 \mathrm{~N}$.m. Fig. 9 illustrates the cogging torque versus mechanical angle. It can be seen that the torque ripple is reduced. After optimization by using level set function, the average radial magnetiv flux density in air gap is remained as $0.31 \mathrm{~T}$.

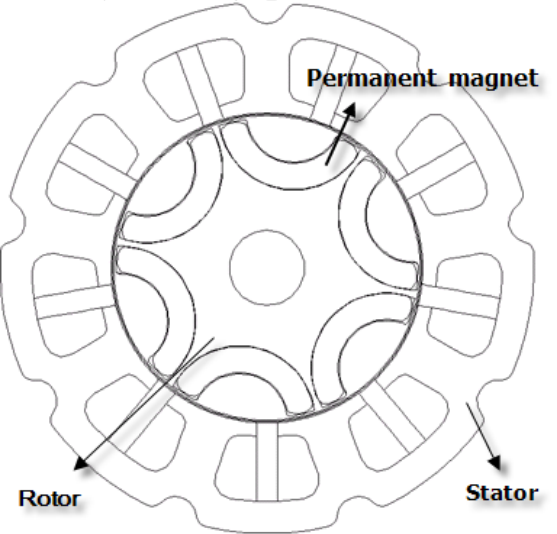

Figure 4: BLDC motor

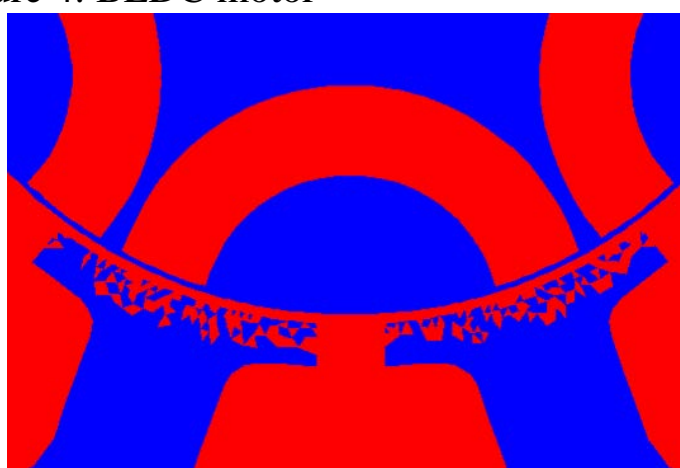

Figure 6: Optimal result with density method

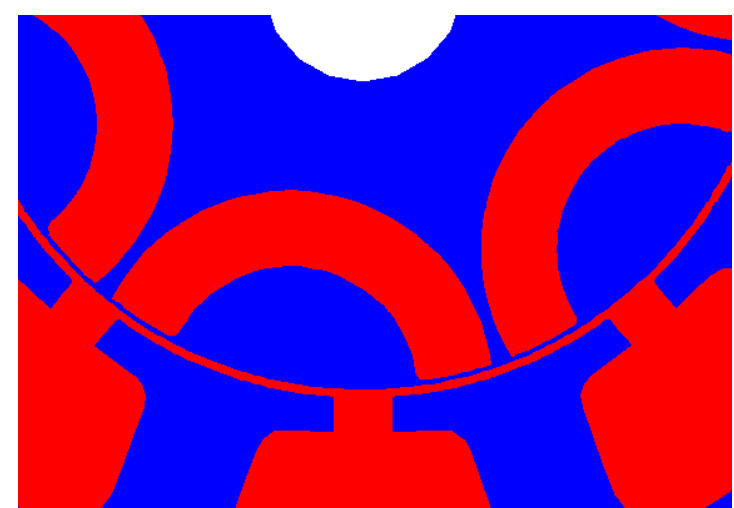

Figure 5: Initial pole shape

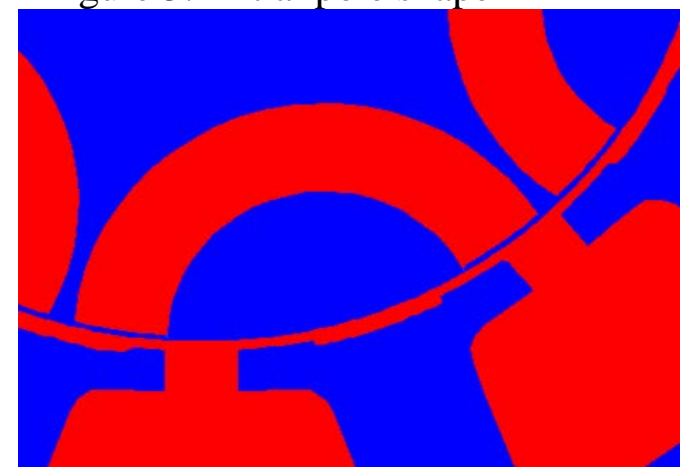

Figure 7: Optimal result with level set 


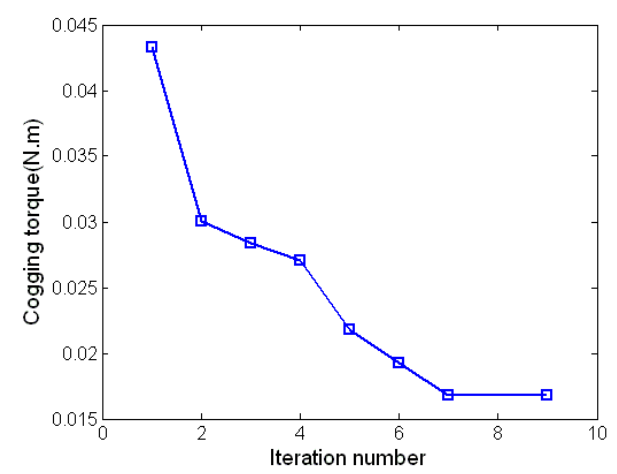

Figure 8: Iteration history

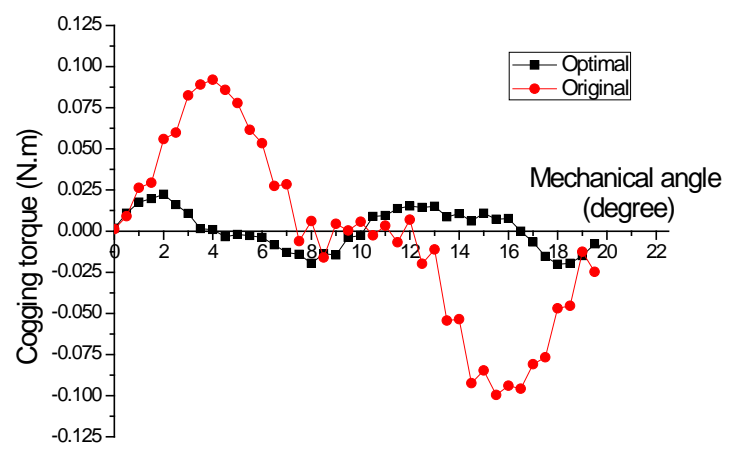

Figure 9: Optimal and original cogging torque

\section{Conclusion}

The level set based topology optimization is presented in this paper. The concept of level set function and adjoint variable method are introduced. The Heaviside function is applied to reluctivity adjustment of the triangular finite elements through which the zero level set boundaries pass. A numerical topology optimization of stator pole shape of a permanent magnet BLDC motor shows that level set based topology optimization may avoid the grayscale elements and achieve smoother boundaries comparing with density method. The ripple of cogging torque can be effectively reduced by using level set based topology optimization.

\section{References}

[1] S.Y. Wang, S.K. Jeong, H. Yoon, Continuum Shape Design Sensitivity Analysis of Magnetostatic Field Using Finite Element Method, IEEE transaction on magnetics. Vol 35, No. 31999 p(1159-1162).

[2] H.K. Shim, S.Y. Wang and K. Lee, 3-D Optimal Design of Induction Motor Used in High-Pressure Scroll Compressor, IEEE transaction on magnetics, Vol. 45, No. 5, March 2009 p(2076-2084).

[3] D.H. Kim, S.B. Lee, et al, Smooth Boundary Topology Optimization for Electrostatic Problems Through the Combination of Shape and Topological Design Sensitivities, IEEE transaction on magnetics, Vol. 44, No. 6, June 2008 p(1002-1005).

[4] N. Takahashi, S. Nakazaki and D. Miyagi, Examination of Optimal Design Method of Electromagnetic Shield Using ON/OFF Method, IEEE transaction on magnetics, Vol. 45, No. 3, March 2009 p(1546-1549).

[5] M.Y. Wang, A level set method for structure topology optimization, Comput. Methods Appl. Mech. Engrg. 192(2003) 227-246.

[6] Z. Luo, M.Y. Wang, et al, A level set-based parameterization method for structural shape and topology optimization, International Journal for numerical methods in engineering Int. J. Numer. Meth. Engng 2008; $76: 1-26$.

[7] E.J. Haug, K.K. Choi and V. Komkov, Design sensitivity analysis of structure systems, Academic Press, New York,1986.

[8] Ole Sigmund, Topology optimization: a tool for the tailoring of structures and materials, Phil. Trans. R. Soc. Lond. A (2000) 358, 211-227

[9] Dong-Kyu Lee, Combined Topology and Shape Optimization of Structures using Nodal Density as Design Parameter, Journal of Asian Architecture and Building Engineering, May 2007166.

[10] Ki-Jin Han, Han-Sam Cho, et al, Optimal Core Shape Design for Cogging Torque Reduction of Brushless DC Motor Using Genetic Algorithm, IEEE transaction on magnetics. Vol 36, No. 42000 p(1927-1931). 